\title{
THE
}

2008

\section{Component-Dependent Fréchet Sensitivity Kernels and Utility of Three-Component Seismic Records}

\author{
Yang Shen \\ University of Rhode Island, yshen@uri.edu \\ Zhigang Zhang \\ University of Rhode Island \\ Li Zhao
}

Follow this and additional works at: https://digitalcommons.uri.edu/gsofacpubs

Terms of Use

All rights reserved under copyright.

\section{Citation/Publisher Attribution}

Yang Shen, Zhigang Zhang, Li Zhao; Component-Dependent Fréchet Sensitivity Kernels and Utility of Three-Component Seismic Records. Bulletin of the Seismological Society of America ; 98 (5): 2517-2525. doi: 10.1785/0120070283

Available at: https://doi.org/10.1785/0120070283

This Article is brought to you for free and open access by the Graduate School of Oceanography at DigitalCommons@URI. It has been accepted for inclusion in Graduate School of Oceanography Faculty Publications by an authorized administrator of DigitalCommons@URI. For more information, please contact digitalcommons-group@uri.edu. 


\title{
Short Note
}

\section{Component-Dependent Fréchet Sensitivity Kernels and Utility of Three-Component Seismic Records}

\author{
by Yang Shen, Zhigang Zhang, and Li Zhao
}

\begin{abstract}
With the exception of shear-wave splitting and receiver function analyses, the phase or amplitude anomaly of a particular arrival is usually measured on only one of the three-component seismic records. Perfectly good waveforms on the other components are often unused. In this article we show that the different components of the same arrival at the same receiver have different travel-time and amplitude sensitivities to variations in the velocity structure. This is a finite-frequency phenomenon for measurements derived from waveforms. It is important where the scales of velocity heterogeneities are comparable or smaller than the width of the Fresnel zone. We calculate the Fréchet sensitivity kernels using the scattering-integral method in conjunction with finite-difference wave simulation in three-dimensional media. The differences in the sensitivity kernels for the different components vary with the wave type, source-receiver geometry, and source mechanism. They are attributed to scattered waves that affect the waveforms on the different components by various amounts. Thus, the differential kernels between the different components of the same arrival may enable us to use the corresponding phase and amplitude measurements, which are relatively accurate observations unaffected by uncertainties in source origin time and location, to image the Earth structure, particularly fine structures near receivers.
\end{abstract}

\section{Introduction}

It has been recognized for some time that seismic waves have finite-frequency bandwidths and are sensitive to a threedimensional (3D) structure off ray paths (e.g., Yomogida, 1992; Friederich et al., 1993; Gudmundsson, 1996; Meier et al., 1997; Marquering et al., 1999; Spetzler et al., 2002; Yoshizawa and Kennett, 2002). In recent years advances in seismic theory have provided theoretical and numerical formulas to calculate the 3D Fréchet sensitivity kernels, the functions that relate the perturbations of the media of wave propagation to seismic observations (e.g., Dahlen et al., 2000; Zhao et al., 2000; Tromp et al., 2005; Zhao et al., 2005). Specifically, phase delays and amplitude anomalies measured by waveform cross correlation are expressed as a volume integration of the product of the perturbations of the media and the Fréchet kernels.

Several methods have been developed to calculate the sensitivity kernels of finite-frequency waves, and there are important differences among the various approaches. Using the Born approximation in conjunction with body-wave ray theory, Dahlen et al. (2000) and Dahlen and Baig (2002) computed the 3D Fréchet sensitivity kernels for finite- frequency body-wave phase delays and amplitude anomalies measured by cross correlation of a broadband waveform with a synthetic seismogram for a spherical (1D) earth. For computational efficiency, forward and like-type scattering from single scatterers and far-field approximations are implemented. The direct $P$ or $S$ body-wave travel-time sensitivity kernels for the one-dimensional (1D) reference earth from these calculations can be characterized as a hollow banana-shaped volume surrounding the ray path. In order to obtain a more accurate and complete wave field in a spherical earth, Zhao et al. (2000) and Zhao and Jordan (2006) employed normal modes in the calculation of the Fréchet kernels. In places where 1D models are inadequate to represent the reference earth, two different computational approaches have been developed to calculate the kernels for 3D reference models: the adjoint (Tromp et al., 2005; Liu and Tromp, 2006) and scattering-integral (Zhao et al., 2005; Zhang et al., 2007) methods. To date, wave propagation in these two approaches is modeled with the spectral element (Komatitsch et al., 2005) and finite-difference (Olsen, 1994) methods, respectively. 
In addition to the fact that finite-frequency kernels more accurately represent the propagation of real seismic waves than ray approximation (Hung et al. 2000; Baig et al., 2003), one of the main reasons that finite-frequency sensitivity kernels have received much attention in the seismological community is that they allow straightforward integration and joint interpretation of data measured at different frequencies and from different phases (body and surface waves). Thus, the sensitivity kernels provide a powerful tool to extract more information about the Earth from broadband seismic records. For the same reason, component-dependent kernels presented herein are important because they allow us to more fully utilize information in three-component seismic records. With the exception of shear-wave splitting and receiver function analyses, the usual practice in seismic data analysis is that a particular arrival is measured on only one of the threecomponent seismic records. For example, the $P$ wave is usually measured on the vertical component, and the $S$ wave is usually measured on the transverse component. Perfectly good waveforms on the other components are often unused. This practice is nonetheless consistent with ray theory, which predicts that the arrival time of a phase is the same on the various components of seismic records at the same station.

In this article we present several examples of the component-dependent Fréchet kernels for finite-frequency seismic waves. The kernels are calculated using the scattering-integral method (Zhao et al. 2005; Zhang et al., 2007) in conjunction with finite-difference wavepropagation calculations (Olsen, 1994; Marcinkovich and Olsen, 2003) in a full-wave approach. We show that the sensitivity kernels for the different components of the same arrival are significantly different, so the usually unused waveforms on the other components contain additional constraints on the Earth structure.

\section{Component-Dependent Sensitivity Kernels}

We calculate the component-dependent sensitivity kernels using a modified scattering-integral method (Zhao et al., 2005). For an arrival within the time window $t_{1}$ and $t_{2}$, we define the travel-time and amplitude anomalies for the component in the direction of a unit vector, $\hat{e}_{l}, \delta \tau_{l}^{p}$ and $\delta \tau_{l}^{q}$, respectively,

$$
\begin{gathered}
\delta \tau_{l}^{p}=-\frac{\int_{t 1}^{t 2} \dot{\tilde{u}}_{l}(t) \delta u_{l}(t) d t}{\int_{t 1}^{t 2}\left|\dot{\tilde{u}}_{l}(t)\right|^{2} d t}, \\
\delta \tau_{l}^{q}=\frac{\int_{t 1}^{t 2} \tilde{u}_{l}(t) \delta u_{l}(t) d t}{\int_{t 1}^{t 2}\left|\tilde{u}_{l}(t)\right|^{2} d t},
\end{gathered}
$$

where $\tilde{u}_{l}(t)$ is the displacement calculated from the reference model in the direction $\hat{e}_{l}, \delta u_{l}(t)$ is the change in the displacement due to a perturbation in the velocity model, and $\dot{\tilde{u}}_{l}(t)$ is the time derivative of the displacement field.
We note that the amplitude anomaly defined here is dimensionless, different from that in Zhao et al. (2005) by a constant. Following the same derivation and after the correction of a typo in Zhao et al. (2005), we have the travel-time and amplitude sensitivities to perturbations in $P$ - and $S$-wave speeds:

$$
\begin{aligned}
K_{l, \alpha}^{p}= & \frac{1}{P_{l}} \int_{t 1}^{t 2} 2 \tilde{\rho} \tilde{\alpha} \dot{\tilde{u}}_{l}\left(\mathbf{r}_{R}, t ; \mathbf{r}_{S}\right) \int_{-\infty}^{\infty} \hat{e}_{l} \\
& \cdot\left[\left(\nabla \cdot \tilde{\mathbf{G}}^{T}\right)(\nabla \cdot \tilde{\mathbf{u}})\right] d \tau d t, \\
K_{l, \alpha}^{q}=- & \frac{1}{Q_{l}} \int_{t 1}^{t 2} 2 \tilde{\rho} \tilde{\alpha} \tilde{u}_{l}\left(\mathbf{r}_{R}, t ; \mathbf{r}_{S}\right) \int_{-\infty}^{\infty} \hat{e}_{l} \\
& \cdot\left[\left(\nabla \cdot \tilde{\mathbf{G}}^{T}\right)(\nabla \cdot \tilde{\mathbf{u}})\right] d \tau d t, \\
K_{l, \beta}^{p}= & \frac{1}{P_{l}} \int_{t 1}^{t 2} 2 \tilde{\rho} \tilde{\beta} \dot{\tilde{u}}_{l}\left(\mathbf{r}_{R}, t ; \mathbf{r}_{S}\right) \int_{-\infty}^{\infty} \hat{e}_{l} \\
& \cdot\left\{(\nabla \tilde{\mathbf{G}})^{213}:\left[(\nabla \tilde{\mathbf{u}})+(\nabla \tilde{\mathbf{u}})^{T}\right]\right. \\
& \left.-2\left(\nabla \cdot \tilde{\mathbf{G}}^{T}\right)(\nabla \cdot \tilde{\mathbf{u}})\right\} d \tau d t, \\
K_{l, \beta}^{q}=- & \frac{1}{Q_{l}} \int_{t 1}^{t 2} 2 \tilde{\rho} \tilde{\beta} \tilde{u}_{l}\left(\mathbf{r}_{R}, t ; \mathbf{r}_{S}\right) \int_{-\infty}^{\infty} \hat{e}_{l} \\
& \cdot\left\{(\nabla \tilde{\mathbf{G}})^{213}:\left[(\nabla \tilde{\mathbf{u}})+(\nabla \tilde{\mathbf{u}})^{T}\right]\right. \\
& \left.-2\left(\nabla \cdot \tilde{\mathbf{G}}^{T}\right)(\nabla \cdot \tilde{\mathbf{u}})\right\} d \tau d t,
\end{aligned}
$$

where $\tilde{\mathbf{G}}$ is $\tilde{\mathbf{G}}\left(\mathbf{r}_{R}, t ; \mathbf{r}\right)$ or $\tilde{\mathbf{G}}\left(\mathbf{r}, t ; \mathbf{r}_{R}\right)$ by reciprocity, the Green's tensor between the receiver at $\mathbf{r}_{R}$ and a specific location in the model $\mathbf{r} ; \tilde{\mathbf{u}}=\tilde{\mathbf{u}}\left(\mathbf{r}, t ; \mathbf{r}_{S}\right)$ is the displacement from the source at $\mathbf{r}_{S}$ to $\mathbf{r}$; and $\tilde{u}_{l}\left(\mathbf{r}_{R}, t ; \mathbf{r}_{S}\right)$ is the displacement from $\mathbf{r}_{S}$ to $\mathbf{r}_{R}$. The superscript $T$ represents the transpose of a tensor. The superscript 213 represents the transposition of the first and the second indices of a thirdorder tensor. $\alpha, \beta$, and $\rho$ are the $P$-wave speed, the $S$-wave speed, and the density, respectively. $P_{l}$ and $Q_{l}$ are the normalization factors for the component in the direction $\hat{e}_{l}$ :

$$
\begin{aligned}
& P_{l}=\int_{t_{1}}^{t_{2}}\left|\dot{\tilde{u}}_{l}(t)\right|^{2} d t, \\
& Q_{l}=\int_{t_{1}}^{t_{2}}\left|\tilde{u}_{l}(t)\right|^{2} d t .
\end{aligned}
$$

The preceding equations show that the sensitivity kernels for the component in the direction $\hat{e}_{l}$ depend on the length of the function of the spatial derivatives of the displacement field $\tilde{\mathbf{u}}$ and the Green's tensors $\tilde{\mathbf{G}}\left(\mathbf{r}, t ; \mathbf{r}_{R}\right)$ in the direction $\hat{e}_{l}$ and the displacement $\tilde{u}_{l}\left(\mathbf{r}_{R}, t ; \mathbf{r}_{S}\right)$. The ker- 
nels thus vary with the wave type, source radiation pattern, and source-receiver geometry.

We use three numerical experiments to illustrate the differences of finite-frequency sensitivity kernels on the different components of seismic records. Starting with the simplest case, we present a 3D homogeneous, isotropic model with an explosive source at depth (Fig. 1a, case 1 in Table 1). The receiver is on the surface at a horizontal distance comparable to the depth of the source, so the direct $P$ wave is recorded on both the vertical and radial components (Fig. 1b). In this and following cases, the radial component of the seismic wave is equivalent to the $x$ component in the local Cartesian coordinate system, the vertical component is equivalent to the $z$ component in the Cartesian coordinate system, and the trans- verse component is equivalent to the $y$ component in the Cartesian coordinate system. The source has a time function

$$
M(t)=\int_{0}^{t} e^{-\alpha\left(t-t_{0}\right)^{2}} d t
$$

where $\alpha$ equals to 20, resulting in a source time function with a dominant period of about $1.2 \mathrm{sec} . t_{0}$ is $0.75 \mathrm{sec}$ in all simulations. The quality factors, $Q$, for $P$ and $S$ waves in the model are set at arbitrarily high values, so we ignore attenuation and focus on the elastic behaviors. The $P$ and $S$ velocities and the parameters of the finite-difference calculation are listed in Table 1. (a)
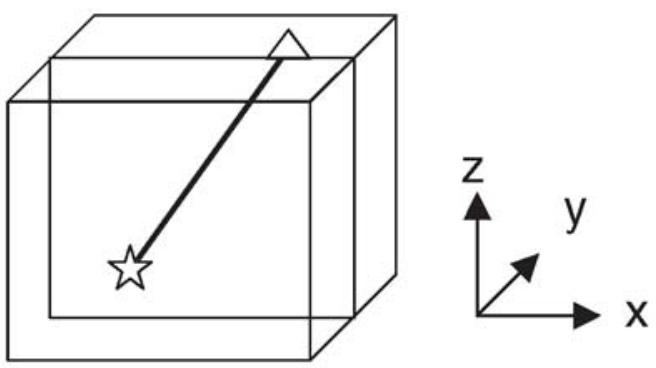

(b)
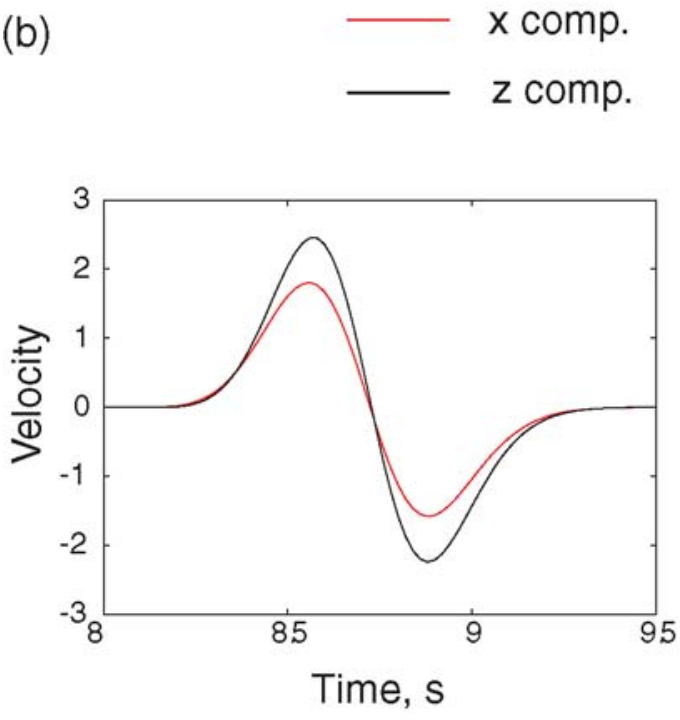

(c)
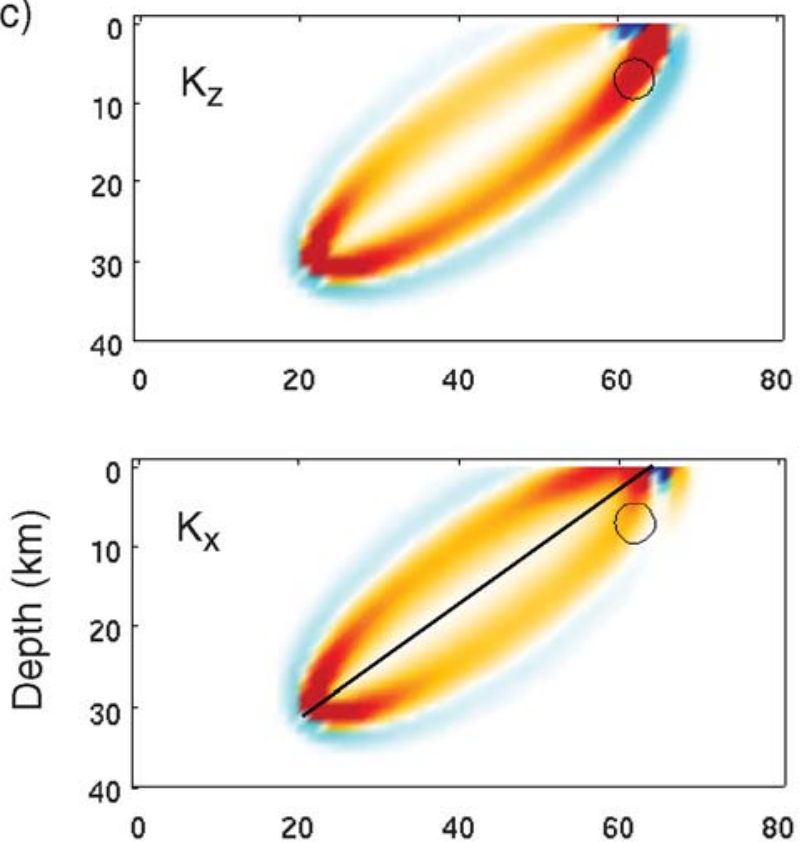

$80 \mathrm{~s} / \mathrm{m}^{3}$

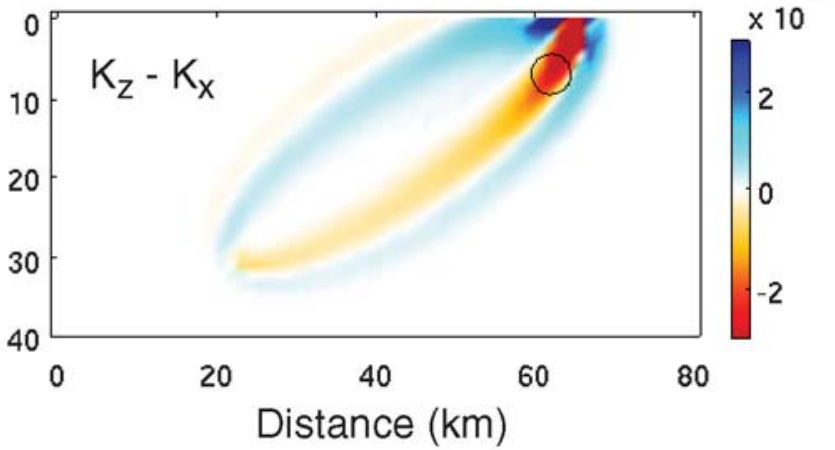

Figure 1. (a) Schematic illustration of a homogeneous, isotropic model with an explosive source at depth (star) and a receiver on the surface (triangle). The directions of the $x, y$, and $z$ coordinate system are marked. (b) The $P$ arrival on the $z$ and $x$ components recorded by the surface receiver. (c) The delay-time kernels for the $z$ and $x$ components and their differential kernel in units of sec $/ \mathrm{m}^{3}$. The negative values (red colors) and positive values (blue colors) are so defined that a low-velocity anomaly located in the region of the negative kernels results in a phase delay, and the same velocity perturbation in the region of positive kernels leads to an earlier arrival as measured by waveform cross correlation. The line represents the ray path from the source (distance, $22.4 \mathrm{~km}$; depth, $30.0 \mathrm{~km}$ ) to the receiver (distance, $64.8 \mathrm{~km}$; depth, $0.0 \mathrm{~km}$ ). The circle shows the location of the spherical, low-velocity anomaly used to test the effects of the scatterer on the waveforms on the different components (Fig. 2). 
Table 1

Parameters of the Models Used in the Numerical Examples

\begin{tabular}{cccc}
\hline & Case 1 & Case 2 & Case 3 \\
\hline Wave type & Direct $P$ & Headwave $(P n)$ & Direct $S(S V, S H)$ \\
Source & Explosive & Explosive & Double couple \\
$V_{P} / V_{S}(\mathrm{~km} / \mathrm{sec})$ & $6.500 / 3.823$ & $5.207 / 3.189$ (layer 1) & $6.500 / 3.823$ \\
& & $9.058 / 5.307$ (layer 2) & \\
Finite-difference grid spacing & $400 \mathrm{~m}$ & $400 \mathrm{~m}$ & $200 \mathrm{~m}$ \\
Finite-difference timestep & $0.02 \mathrm{sec}$ & $0.02 \mathrm{sec}$ & $0.015 \mathrm{sec}$ \\
\hline
\end{tabular}

Figure 1c shows the travel-time sensitivities to $P$-velocity perturbations for the $z$ and $x$ components and their differential kernels. The differential kernels are relatively large near the receiver (Fig. 1c) and become smaller in magnitude away from the receiver. Near the receiver and below the ray path, the sensitivities for the $z$-component record are much stronger than for the $x$-component record. On the other hand, the sensitivities are much stronger for the $x$ component above the ray path near the receiver. The differences in the sensitivities between the different components can be understood by imagining a scatterer in the model. For a scatterer near and beneath the receiver, the geometry between the scatterer and the receiver and the nature of $P$-wave particle motion determine that most of the scattered $P$-wave energy is on the $z$ component, therefore affecting the vertical waveform and delay time more strongly than the horizontal waveform and delay time. Similarly, the scattered energy from a scatterer at a shallow depth near the receiver and above the ray path is mostly on the $x$ component, affecting more strongly the $x$-component waveform and delay time. $\mathrm{Wu}$ and Aki (1985) and Favier et al. (2004) provide extensive discussions on the characteristics of the scattered waves by elastic heterogeneities.

To validate this interpretation, we place a spherical perturbation with a $P$-velocity reduction of $4 \%$ and a radius of $2.5 \mathrm{~km}$ below the ray path near the receiver, as shown by the circle in Figure 1c. All other elastic properties of the model remain the same. Because delay time is the volume integration of the product of the kernels and the velocity perturbation in the finite-frequency formulation, the componentdependent kernels in Figure 1c predict a larger delay for the $z$ component than for the $x$ component. Figure 2 shows the normalized $P$ waveforms for the models with and without the spherical perturbation. The waveform change on the $z$ component caused by the spherical perturbation is significantly larger than on the $x$ component, and the delay on the vertical component measured by cross correlation of the waveforms with and without the velocity perturbation ( $\Delta t=0.009 \mathrm{sec}$ ) is three times larger than the delay on the horizontal component $(\Delta t=0.003 \mathrm{sec})$. The crosscorrelation measurements are consistent quantitatively with the predicted values from the component-dependent kernels ( 0.008 and $0.003 \mathrm{sec}$ for the $z$ and $x$ components, respectively), within small numerical and round-off errors.
To illustrate how the incidence angle of the arrival affects the sensitivity kernels, we adjust the horizontal distance between the receiver and source. Figure 3 shows a sourcereceiver geometry that results in a direct arrival with a steep incident angle and, thus, a larger amplitude on the vertical component than on the horizontal component. Compared to
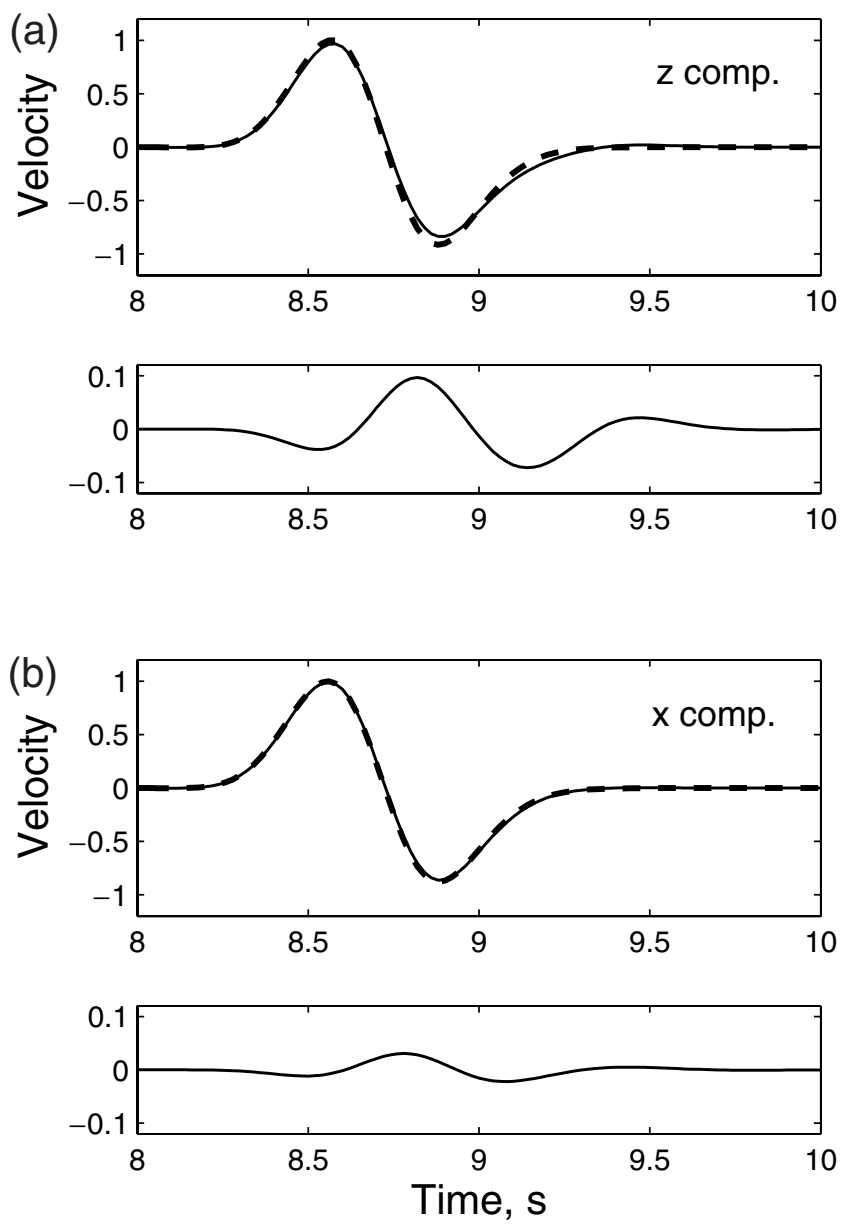

Figure 2. The normalized $P$ waveforms in particle velocity at the receiver for the models with (thin line) and without (thick dashed line) the velocity perturbation in case 1 (Fig. 1). (a) The top panel shows the $P$ waveforms on the $z$ component. The second panel is the difference between the waveforms for the models with and without the velocity perturbation, shown at an enlarged vertical scale. (b) The description is the same as for part (a) but for the $x$ component. 
travel time
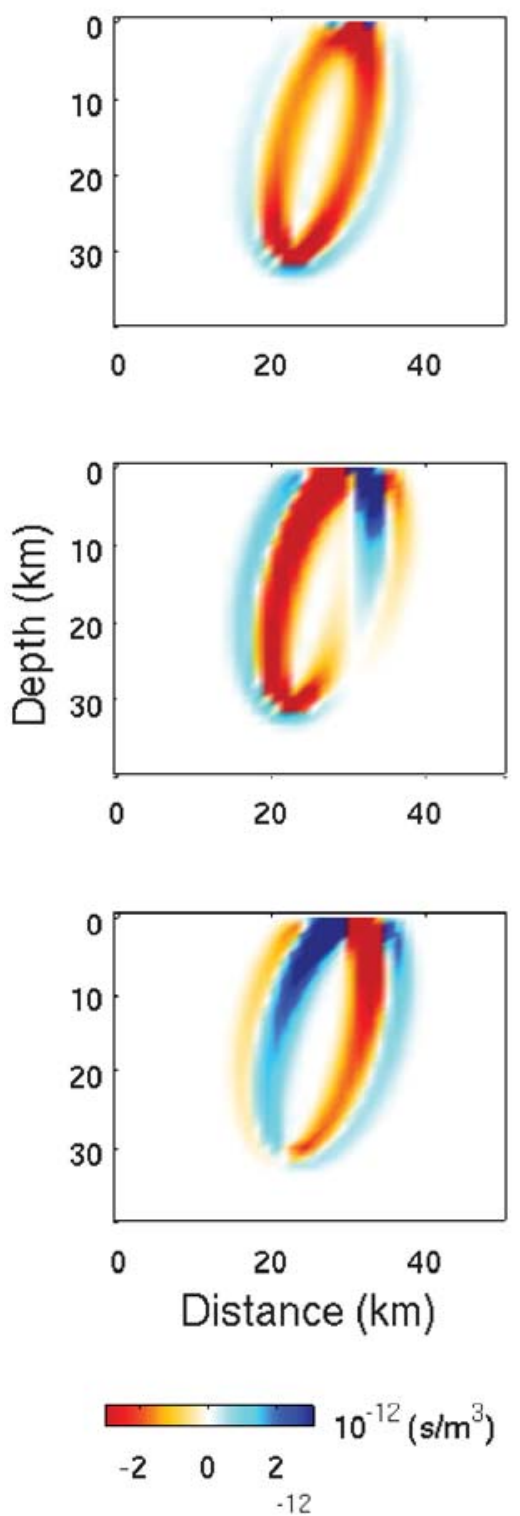

amplitude

$\mathrm{K}_{\mathrm{z}}$

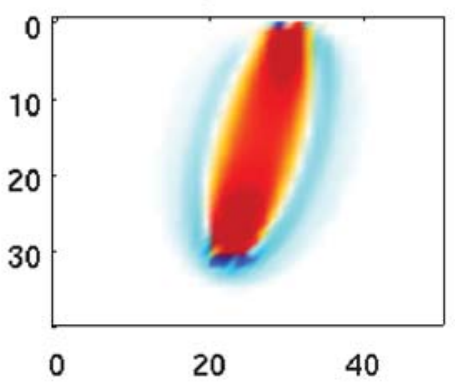

$\mathrm{K}_{\mathrm{x}}$

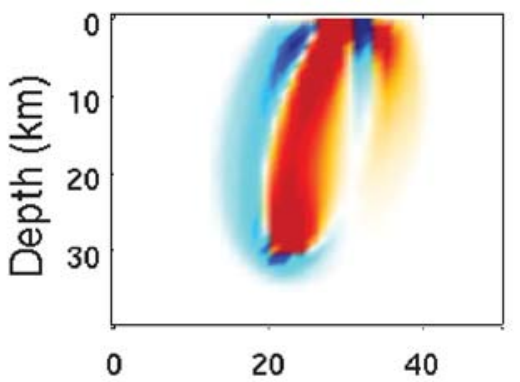

$\mathrm{K}_{\mathrm{z}}-\mathrm{K}_{\mathrm{x}}$
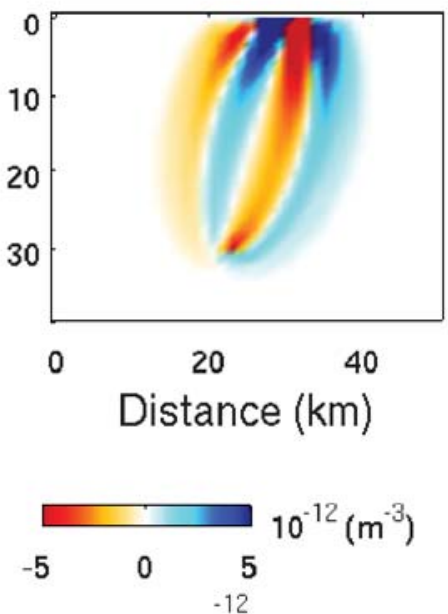

Figure 3. Delay-time (left-hand side) and amplitude (right-hand side) sensitivity kernels for the $z$ and $x$ components and their differential kernel. The surface receiver $(30.8 \mathrm{~km}, 0)$ is at a relatively short horizontal distance from the explosive source at depth $(22.8,30.0 \mathrm{~km})$. The travel-time kernels are defined in the same way as in Figure 1c. For amplitude, the negative values (red colors) and positive values (blue colors) are so defined that a high-velocity anomaly located in the region of the negative kernels results in an amplitude reduction, and the same velocity perturbation in the region of positive kernels leads to an increase in amplitude.

Figure 1c, the travel-time kernels for the $x$ component show a greater asymmetry about the ray path between the source and receiver. Figure 3 also shows, as an example, the componentdependent amplitude kernels. Like the travel-time kernels, the amplitude kernels for the $x$ component also show a great degree of asymmetry about the ray path. This asymmetry is due to the fact that the propagation direction of the horizontal component of a scattered wave from a heterogeneity to the far side of the receiver is in the opposite direction of the $x$ component of the direct wave from the source to the receiver, while the horizontal component of a scattered wave from a heterogeneity to the near side of the receiver is in the same direction of the $x$ component of the direct wave. The opposite effects of the scattered wave on the waveforms at the receiver result in the opposite polarities of the $x$-component kernels about the receiver. Furthermore, the sensitivity for the $x$ component is a minimum (zero) directly beneath the receiver, reflecting the fact that a $P$ wave bounced off a scatterer directly beneath the receiver propagates vertically to the receiver and thus does not affect the waveform (nor the delay time and amplitude) on the $x$ component. The differential kernels are more pronounced than in Figure 1c. 
For a receiver directly above the source, the $P$ arrival has no energy on the $x$ component in this homogeneous and isotropic reference model. Without a reference waveform, the kernel calculation for the $x$ component becomes unstable ( $P_{l}$ and $Q_{l}$ in equations 7 and 8 are zero). In practice, when the signal of the arrival on one of the components is below the noise level, that component does not yield useful delaytime and amplitude measurements.

In case 2, we introduce one layer over a higher-velocity half-space. Within the layer and the half-space, the velocities are uniform (Table 1). We use the same explosive source as in case 1 but examine the sensitivity kernels for the headwave instead (Fig. 4). In order to investigate the effects of the scatterers near the receiver in a different setting, we place the receiver below the surface. Because the velocity in the halfspace is uniform, the downward bending of the kernels in the half-space is attributed to the increasing Fresnel-zone width from the source and receiver and has nothing to do with a vertical velocity gradient (Zhang et al., 2007). Again the differences between the travel-time sensitivity kernels for the $z$ and $x$ components are pronounced. Below the layer interface and near the receiver, the sensitivity for the $z$ component is stronger in absolute values than for the $x$ component at the
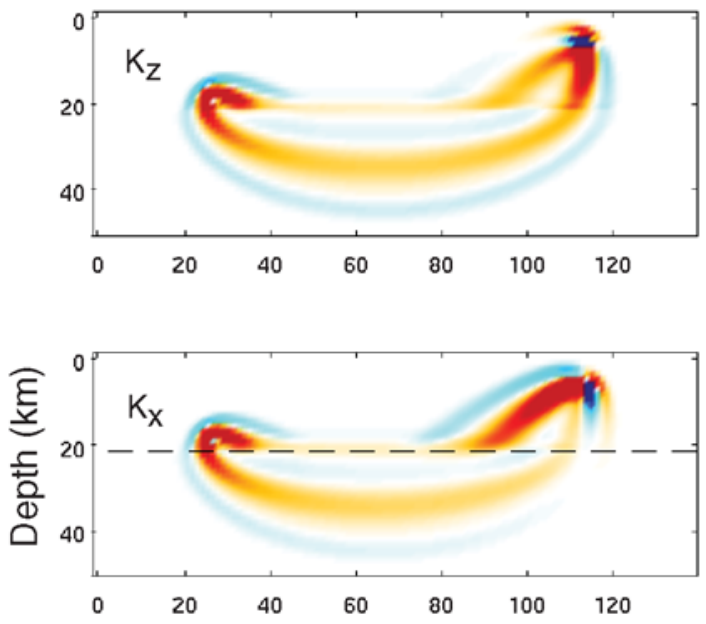

$\mathrm{s} / \mathrm{m}^{3}$

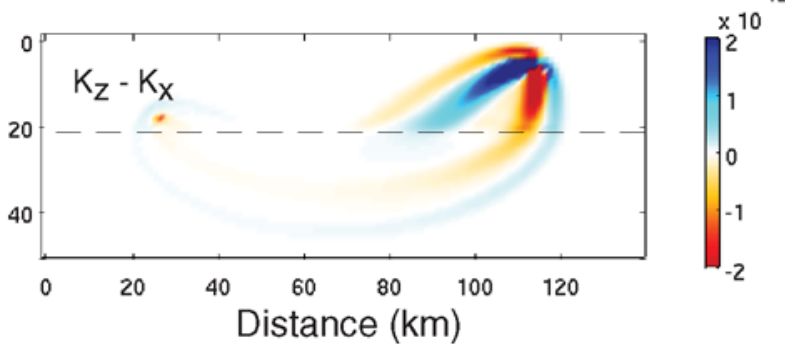

Figure 4. Headwave delay-time sensitivity kernels for the $z$ and $x$ components and their differential kernel. The dashed line marks the interface between the top layer and a higher-velocity half-space. The source is located on the left-hand side of the model (26.0, $18.0 \mathrm{~km})$. The receiver is near the surface $(113.2,6.4 \mathrm{~km})$. The negative values (red colors) and positive values (blue colors) are defined in the same way as in Figure 1c. same location. Above the interface and to the source side of the receiver, the sensitivity for the $x$ component is stronger than for the $z$ component. The differences can also be explained in the same way as for the direct $P$ arrival. Depending on the location of the scatterer, the scattered energy is mostly on one or the other component and therefore affects the waveforms and delay times on the two components unevenly. Numerical experiments with a spherical $P$-velocity perturbation, similar to that in case 1 , again validate this interpretation of the headwave kernels (not shown). However, unlike case 1 , the differential kernels are very small near the source. The lack of differential kernels near the source can be explained by the fact that away from the receiver only the scattered waves that propagate (sub-) horizontally below the interface arrive within the time window of the headwave and contribute to the calculation of the headwave kernels. Consequently, scattered waves from velocity perturbations near the source do not disproportionally perturb the $z$ and $x$ components of the headwave and cause differential kernels.

Case 3 has a double-couple source with a strike of $0^{\circ}$, a dip of $30^{\circ}$, and a rake of $45^{\circ}$ in a homogeneous and isotropic model. The strike of $0^{\circ}$ is defined as the direction of the $y$ axis in the local coordinate. The source-receiver geometry is similar to that in case 1 (Fig. 1) except that the source is at $40 \mathrm{~km}$ depth and the receiver is $(7 \mathrm{~km})$ below the surface. The $S V$ wave is recorded on the $x$ and $z$ components. The $S H$ wave is recorded on the $y$ component. Figure 5 is a comparison of the $S$ travel-time sensitivities to $S$-velocity perturbations for the three components at three depths: 7,15 , and $25 \mathrm{~km}$. There are obvious differences in the kernels for the different components, though it is difficult to separate and visualize the individual contributions of the various scattered waves in this double-couple case because of $S V$ to $P, S V$, and $S H$ scattering and $S H$ to $P, S V$, and $S H$ scattering (Wu and Aki, 1985; Dahlen et al., 2000). Also complicating the picture are the radiation patterns of $S V$ and $S H$ waves for the double-couple source. But it is still straightforward to validate the component dependence of the $S$-wave kernels numerically. We put a spherical anomaly with an $S$-velocity reduction of $4 \%$ and a radius of $3.0 \mathrm{~km}$ at $15 \mathrm{~km}$ depth as shown by the circle in Figure 5. The kernels predict that the resultant $S$ delays on the $z$ component should be larger than on the $x$ and $y$ components. The waveform changes on the three components caused by the velocity perturbation are shown in Figure 6. Indeed the perturbed waveform is most pronounced on the $z$ component. The delay time on the vertical component from the cross correlation of the reference and perturbed waveforms $(\Delta t=0.019 \mathrm{sec})$ is about twice as large as the delay time on the $x$ component $(\Delta t=0.009 \mathrm{sec})$, while the delay time on the $y$ component $(\Delta t=0.015 \mathrm{sec})$ is about $50 \%$ larger than on the $x$ component, again consistent with the kernel predictions $(0.018,0.009$, and $0.014 \mathrm{sec}$ for the $z$, $x$, and $y$ components, respectively).

While the ratios of the travel-time anomalies on the different components are large, the absolute differential delays calculated previously are likely smaller than observed 


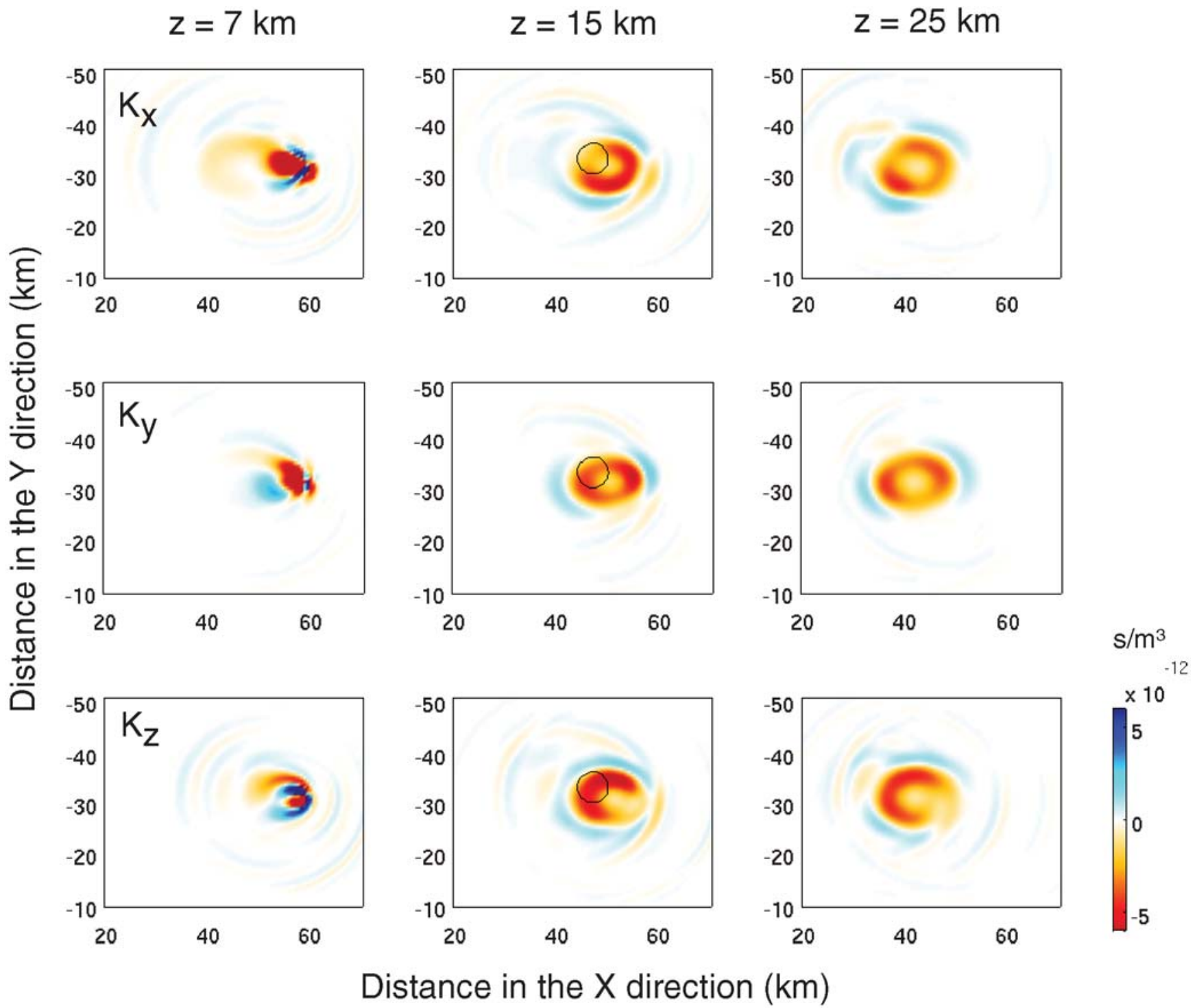

Figure 5. Comparison of the travel-time sensitivities to $S$-velocity perturbations for the $x, y$, and $z$ components of the direct $S$ wave in a homogeneous and isotropic model (case 3$)$. The source and receiver locations in kilometers and the $x, y$, and $z$ directions are $(27.8,31.2,40.0)$ and $(58.6,31.2,7.0)$, respectively. The top row shows the kernels for the $x$ component at 7 (the depth of the receiver), 15 , and $25 \mathrm{~km}$. The middle and bottom rows are for the $y$ and $z$ components, respectively. The negative values (red colors) and positive values (blue colors) are defined in the same way as in Figure 1c. The circles mark the location of the spherical $S$-velocity perturbation used to test the effect of the scatterer on the $S$ waveforms on the different components (Fig. 6).

travel-time anomalies in real seismic experiments. The small travel-time delays are the direct results of the relatively small volume of the input spherical velocity perturbation. When a much larger volume of the velocity heterogeneity is located in places where the differential kernels (Figs. 1 and 3) are strong, it can cause much greater differential delays on the different components.

\section{Conclusions}

We demonstrate that the finite-frequency Fréchet kernels calculated from a full-wave method can be significantly different for the different components of the same arrival at a receiver. The differences in the kernels are attributed to the scattered waves that affect the different components of seismic records unequally and suggest that a seismic arrival on more than one component of the three-component records can be utilized to constrain the Earth structure. This component dependence of the sensitivity kernels is a finitefrequency phenomenon for measurements derived from waveforms. It is important where the scales of velocity heterogeneities are comparable or smaller than the sensitivity regions (Figs. 1, 3, 4 and 5). As a rule of thumb, the size of the sensitivity region can be approximated by the full width of the first plane-wave Fresnel zone $\left(\sqrt{4 \lambda L+\lambda^{2}}\right.$ or $\sim 2 \sqrt{\lambda L}$ when $L \gg \lambda$, where $\lambda$ is the wavelength and $L$ the distance 
(a)
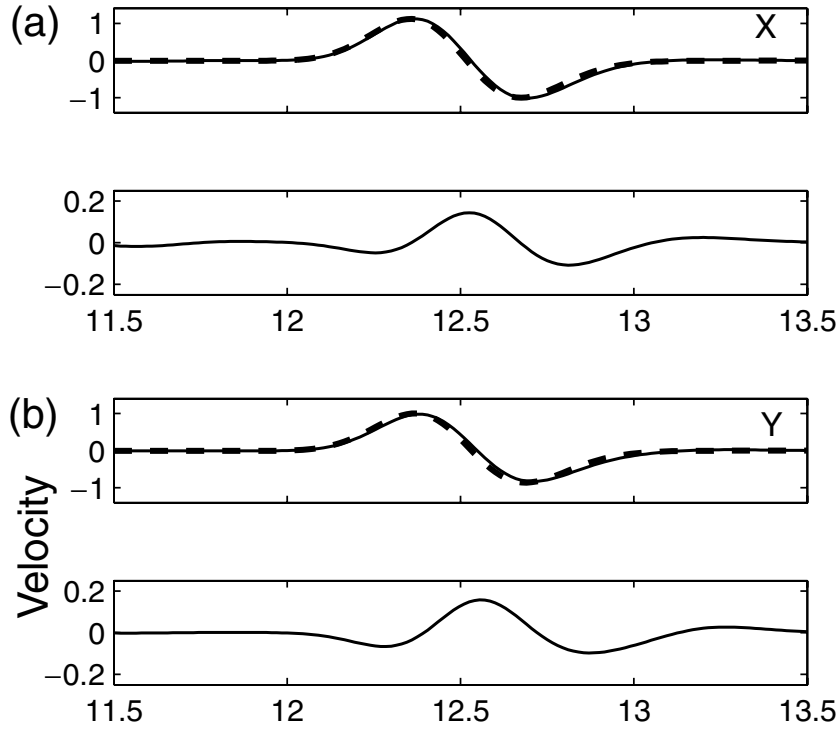

(c)
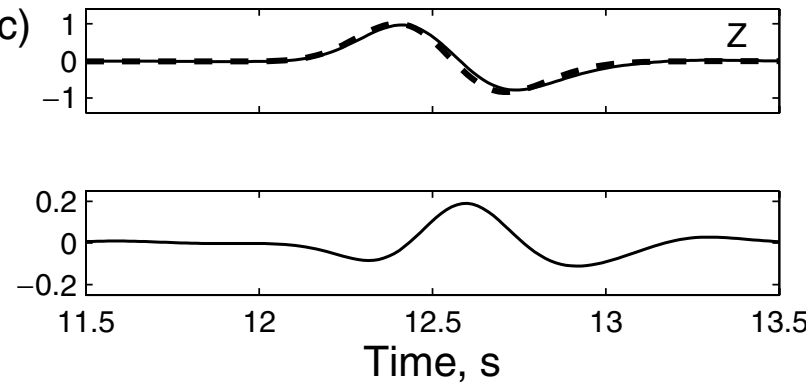

Figure 6. The normalized $S$ waveforms in particle velocity at the receiver for the models with (thin line) and without (thick dashed line) the spherical velocity perturbation in case 3 (Fig. 5). (a) The top panel shows the $S$ waveforms on the $x$ component, reversed in polarity to aid the visual comparison to the other components. The second panel is the difference between the waveforms for the models with and without the velocity perturbation. (b) The same description as for (a) but for the $y$ component. (c) The same description as for (a) but for the $z$ component.

to the receiver). For scale, a $20 \sec P$-wave propagating near vertically at $400 \mathrm{~km}$ depth to a surface receiver has a full Fresnel-zone width of $\sim 570 \mathrm{~km}$. As wave frequency increases, the size of the Fresnel zone decreases, and the component dependence of the sensitivity kernels becomes less important for broad and smooth velocity heterogeneities. Nevertheless as one can see from geological outcrops, strong heterogeneities may exist at many length scales down to the size of hand samples. Because the differential travel-time and amplitude measurements between the different components of the same arrival are not affected by uncertainties in earthquake origin time and, in some cases, source location errors, the differential travel-time and amplitude measurements may be particularly useful for imaging the fine structure near receivers. Finally, the component-dependent sensitivity kernels suggest that caution should be practiced in the interpretation of shear-wave splitting in places where heterogeneities are strong and have dimensions that are comparable to the width of the sensitivity kernels, as both intrinsic anisotropy and isotropic heterogeneities may cause an apparent travel-time difference on two components of an $S$ wave.

\section{Data and Resources}

No observational data were used in this article. The calculations were carried out on a nine-node Linux PC cluster at the Graduate School of Oceanography, University of Rhode Island.

\section{Acknowledgments}

This work was supported by the U.S. National Science Foundation (Grant Number NSF-OCE 0425747) and by the U.S. Air Force Research Laboratory (Contract Number fa8718-06-C-0014). We thank Qinya Liu and an associate editor for their thoughtful and helpful comments.

\section{References}

Baig, A. M., F. A. Dahlen, and S.-H. Hung (2003). Traveltimes of waves in three-dimensional random media, Geophys. J. Int. 153, 467-482.

Dahlen, F. A., and A. M. Baig (2002). Fréchet kernels for body-wave amplitudes, Geophys. J. Int. 150, 440-466.

Dahlen, F. A., S.-H. Hung, and G. Nolet (2000). Fréchet kernels for finite frequency traveltimes, I. Theory, Geophys. J. Int. 141, 157-174.

Favier, N., S. Chevrot, and D. Komatitsch (2004). Near-field influence on shear wave splitting and traveltime sensitivity kernels, Geophys. J. Int. 156, 467-482.

Friederich, W., E. Wielandt, and S. Strange (1993). Multiple forward scattering of surfacve waves: comparison with an exact solution and the Born single-scattering methods, Geophys. J. Int. 112, 264-275.

Gudmundsson, O. (1996). On the effect of diffraction on traveltime measurements, Geophys. J. Int. 124, 304-314.

Hung, S.-H., F. A. Dahlen, and G. Nolet (2000). Fréchet kernels for finite frequency traveltimes, II. Examples, Geophys. J. Int. 141, 175-203.

Komatitsch, D., S. Tsuboi, and J. Tromp (2005). The spectral-element method in seismology, in Seismic Earth: Array Analysis of Broadband Seismograms, A. Levander and G. Nolet (Editors), Geophysical Monograph 157, 205-227.

Liu, Q., and J. Tromp (2006). Finite-frequency kernels based on adjoint methods, Bull. Seismol. Soc. Am. 96, 2383-2397.

Marcinkovich, C., and K. B. Olsen (2003). On the implementation of perfectly matched layers in the fourth-order velocity-stress finitedifference scheme, J. Geophys. Res. 108, 2276, doi 10.1029/ 2002JB002235.

Marquering, H., F. A. Dahlen, and G. Nolet (1999). Three-dimensional sensitivity kernels for finite-frequency traveltimes: the banana-doughnut paradox, Geophys. J. Int. 137, 805-815.

Meier, T., S. Lebedev, G. Nolet, and F. A. Dahlen (1997). Diffraction tomography using multimode surface waves, J. Geophys. Res. 102, $8255-8267$.

Olsen, K. B. (1994). Simulation of three-dimensional wave propagation in the Salt Lake Basin, Ph.D. Thesis, University of Utah, Salt Lake City, Utah, 157 pp.

Spetzler, J., J. Trampert, and R. Snieder (2002). The effect of scattering in surface wave tomography, Geophys. J. Int. 149, 755-767.

Tromp, J., C. Tape, and Q. Liu (2005). Seismic tomography, adjoint methods, time reversal and banana-doughnut kernels, Geophys. J. Int. 160, 195-216.

Wu, R., and K. Aki (1985). Scattering characteristics of elastic waves by an elastic heterogeneity, Geophysics 50, 582-595.

Yomogida, K. (1992). Fresnel-zone inversion for lateral heterogeneities in the Earth, Pure Appl. Geophys. 138, 391-406. 
Yoshizawa, K., and B. L. N. Kennett (2002). Determination of the influence zone for surface wave paths, Geophys. J. Int. 149, 440-453.

Zhang, Z., Y. Shen, and L. Zhao (2007). Finite-frequency sensitivity kernels for head waves, Geophys. J. Int. 171, 847-856.

Zhao, L., and T. H. Jordan (2006). Structural sensitivities of finite-frequency seismic waves: a full-wave approach, Geophys. J. Int. 165, 981-990.

Zhao, L., T. H. Jordan, and C. H. Chapman (2000). Three-dimensional Fréchet differential kernels for seismic delay times, Geophys, J. Int. 141, $558-576$.

Zhao, L., T. H. Jordan, K. B. Olsen, and P. Chen (2005). Fréchet kernels for imaging regional earth structure based on three-dimensional reference models, Bull. Seismol. Soc. Am. 95, 2066-2080.
Graduate School of Oceanography

University of Rhode Island

Narragansett, Rhode Island 02882

yshen@gso.uri.edu

(Y.S., Z.Z.)

Institute of Earth Sciences

Academia Sinica

Taipei 115, Taiwan

(L.Z.)

Manuscript received 19 November 2007 